\title{
Gestión Financiera y Competitividad. Estudio de las MiPymes de la ciudad Machala
}

\author{
Financial management and Competitiveness. Study \\ of Machala city MSMES
}

Alonso-Aguilar Daniela Universidad de Vigo - España falonso@uvigo.es

Chavez-Mora Miguel Universidad de Vigo - España pchavez@uvigo.es

Revista Cumbres Vol.7 №1

Versión electrónica ISSN 1390-3365 http://investigacion.utmachala.edu.ec/revistas/index.php/Cumbres http://doi.org/10.48190/cumbres.v7n1a2 


\title{
RESUMEN
}

Sin importar el tamaño de una empresa o su actividad económica, las instituciones tienen como objetivo maximizar sus ganancias, surgiendo la necesidad de ser competitivas; a pesar de que la competitividad no es un concepto estático, sino que se alcanza con la sinergia de una serie de factores de tipo económico-social, en este estudio se ha enfocado en uno de esos factores: La gestión financiera; El objetivo del presente trabajo es analizar la forma en la que las micro, pequeñas y medianas empresas de la ciudad de Machala administran sus recursos financieros, y con estos resultados se elaborarán recomendaciones que permitan a estas organizaciones ser competitivas. Para ejecutar esta investigación se realizó 252 encuestas empresas del grupo de las MiPymes, y tras un análisis del mismo se llegó a la conclusión que la competitividad está estrechamente relacionada con la gestión financiera, ahorro y gestión adecuada de los recursos.

Palabras clave: gestión financiera, Competitividad, Mipymes de Machala, Finanzas, Tasas de Interés.

\begin{abstract}
Regardless of the size of a company or its economic activity, the institutions aim to maximize their profits, arising the need to be competitive; Although competitiveness is not a static concept, but is achieved with the synergy of a series of economic-social factors, this study has focused on one of these factors: Financial management; The objective of this paper is to analyze the way in which micro, small and medium-sized companies in the city of Machala manage their financial resources, and with these results recommendations will be made that allow these organizations to be competitive. To carry out this research, 252 company surveys of the MSMEs group were carried out, and after an analysis of the same it was concluded that competitiveness is closely related to financial management, savings and adequate management of resources.
\end{abstract}

Keywords: financial management, Competitiveness, MSMES de Machala, Finance, Interest Rates. 


\section{INTRODUCCIÓN}

Frente a un mercado globalizado y exigente, es difícil encontrar un ámbito empresarial en el que no se hable constantemente de "competitividad"; no está por demás explicar este concepto visto desde la perspectiva empresarial tiene su origen tres siglos atrás, cuando en 1776 Adam Smith formuló el concepto de "ventaja absoluta"; años después (Smith, 2011), en 1817 David Ricardo expuso su teoría de la "ventaja comparativa" donde se incorporan temas de especialización, internacionalización y economía a escala (Ricardo, 2003); y finalmente en 1980 Michael Porter desarrolla el concepto de "ventaja competitiva", el cual se basa en que una empresa es competitiva cuando logra una posición superior que las demás empresas que compiten en el mercado (Porter, 2017).

Hoy en día, se puede decir que una empresa es competitiva, cuando con igual o menor inversión obtiene mayor rentabilidad que la competencia en el mismo periodo de tiempo (Corporación Universidad de Asturias, 2014; Sánchez-Gutiérrez, Cabanelas, Lampón, y González-Alvarado, 2019); esto es posible si se cumple al menos una de las siguientes condiciones:

1. Debe aumentar la eficiencia de sus procesos productivos y administrativos, para reducir sus costos, y poder competir en el mercado por precio (Bermeo y Bermeo, 2005); o

2. Dar valor agregado a su producto/servicio mediante la percepción de calidad, imagen o marca, para poder aumentar sus costos y aumentar las ganancias relativas (Deming, 2018; Sánchez-Gutiérrez et al, 2019).

Existe un vínculo inseparable entre la competitividad y la gestión financiera (Solleiro y Castañon, 2005; Suñol, 2006; y Aragón, 2007; Saavedra, Milla y Tapia 2013), para que una empresa alcance la competitividad es necesario que invierta en: infraestructura, recursos humanos y tecnología (Staníčková y Melecký, 2020), y la organización que no sea capaz de financiarlos difícilmente podrá ser competitiva (Liu, 2017).

Con este preámbulo, el presente trabajo investigativo trata de determinar la relación entre la competitividad empresarial y la gestión financiera; a través de un modelo relacional en donde sea posible evidenciar las prácticas realizadas por las empresas más competitivas, considerando así a aquellas que hayan obtenido una mayor rentabilidad.

\section{Gestión financiera}

Hoy en día es posible encontrar diferentes conceptos, y apreciaciones sobre la administración de las finanzas de una empresa (Águila y Tolamatl-Michcol, 2016); sin embargo, esta información se podría resumir en que la Gestión Financiera se basa en la administración adecuada de los recursos empresariales, mediante el control de ingresos y gastos, sumados a una adecuada planificación (Azeez, Kadhim y Kadhim, 2020) y previsión de las necesidades de la organización. 
Un aspecto importante de la gestión financiera de una institución es la necesidad de una constante evaluación que permita conocer la situación real y actualizada de una empresa, y con base en esa información se tome decisiones (Jewell, Reading, Clarke y Kippist, 2020), los aspectos en los que se debe poner atención son:

- Situación contable y tributaria

- Valoración financiera

- Endeudamiento

- Ahorro

\section{Situación contable y tributaria}

La contabilidad dentro de una empresa, es un proceso que permite a los empresarios, conocer la situación patrimonial de la misma (Karlsson, 2019), indistintamente del tamaño de la empresa, es necesario llevar un control minucioso y detallado de las operaciones de ingresos y egresos, que permita estar al tanto de la disponibilidad de recursos de la empresa; y una vez se haya llevado a cabo ese control, es obligatorio reportarlo al ente regulador de tributación en nuestro caso al Servicio de Rentas Internas, para evitar sanciones (LORTI, 2004).

\section{Valoración financiera}

El ratio financiero Rentabilidad Sobre Recursos Propios conocido también como ROE por sus siglas en inglés "Return on Equity" es el aspecto más significativo al momento de evaluar la inversión o realizar análisis comparativos entre las distintas empresas (Kwak, Kim y Yoo, 2011); pues si bien es cierto existen muchas maneras de medir la competitividad de las empresas, ninguno es tan efectivo como medir la rentabilidad (Porter, 2008; Choi, 2021); para ello se utiliza la siguiente fórmula:

Ecuación 1

$$
\text { ROE }=\frac{\text { Rentabilidad }}{\text { Patrimonio }}
$$

Fuente: Ha \& Pham, 2021

En concordancia con el estudio, considerando que la tasa referencial para microcrédito minorista oscila entre $21.52 \%$ y 30,50\% (BCE, 2021) los autores considerarán que una empresa será competitiva cuando tenga un ROE mínimo del $40 \%$.

\section{Cumbres}




\title{
Endeudamiento
}

En América Latina el tema del endeudamiento despierta opiniones encontradas, entre quienes creen que la financiación bancaria es positiva y contribuye con el crecimiento económico (Haro, Galvez, Saez, \& Caba, 2017) y quienes por razones culturales tienen aversión hacia la deuda motivados por el temor de no poder hacer frente a las obligaciones contraídas (Pacheco, 2015); en el caso específico del Ecuador, este rechazo es más visible debido a las elevadas tasas de interés (Banco Central del Ecuador, 2020) y a los costos financieros que conlleva la apertura de líneas de crédito (Campion, Ekka, \& Wenner, 2012).

Indistintamente del tema cultural, es necesario reconocer que el acceso a créditos permite a las empresas apalancarse para financiar su crecimiento (Rivera, 2002), a través de la adquisición de tecnología, herramientas y equipos, que permita al empresario ser más eficiente; además de la posibilidad de deducir los costos financieros de las utilidades anuales, y reducir el impacto de los impuestos (Modigliani y Miller, 1963), siempre que los costes de crédito no sean excesivos, y la proporción de exigible a corto plazo, para medir esta última, se aplica la siguiente ecuación:

Ecuación 2

$$
\text { Calidad de la deuda }=\frac{\text { Pasivos Circulantes }}{\text { Pasivos Totales }}
$$

Fuente: Alarcón y Ulloa (2012)

Desde el punto de vista financiero no es recomendable que el total de las deudas tengan vencimiento en el mismo ejercicio, esta situación puede ocasionar presión innecesaria sobre la empresa (Susanto y Meiryani, 2019), y obligar a que la gerencia adquiera nueva deuda para hacer frente a obligaciones próximas a vencerse, aumentando el costo financiero.

\begin{abstract}
Ahorro
A razón de que ninguna actividad económica está exenta de los imprevistos, siniestros o cambios en el mercado (Dzhalilova y Meza-Godoy, 2020), ahorrar se constituye en uno de los pilares fundamentales para el crecimiento empresarial y el cumplimiento de las metas y objetivos institucionales (García-Magaña y Vargas-Hernández, 2014). En Ecuador, el ahorro es obligatorio, la Ley de Compañías en su artículo 297 establece que las sociedades deben realizar una reserva no menor al $10 \%$ de sus utilidades líquidas al finalizar cada ejercicio económico, el cual está destinado al fondo de reserva anual, el cual debe acumularse hasta alcanzar al 50\% del capital social (Ley de Compañías, 2014), sin embargo, esto es aplicable solo a las personas jurídicas, las personas naturales tienen albedrío para la distribución de ganancias.
\end{abstract}




\section{MATERIALES Y METODOLOGÍA}

Para realizar la fundamentación teórica de este trabajo se recurrió a la revisión exhaustiva de la literatura en revistas de alto impacto, además, se realizó una encuesta a 252 empresarios pertenecientes a las MiPymes con el propósito de contrastar la información recopilada, el cuestionario recogió datos referentes a: entorno general de las empresas, situación contable y tributaria, inversión requerida, ingresos anuales, historial crediticio, niveles de endeudamiento y ahorro; los participantes fueron escogidos por muestreo aleatorio simple; la información fue procesada por medio del software de análisis estadístico RCommander.

Para el alcance del objetivo de investigación se diseñó un modelo relacional que permita determinar la relación entre la competitividad empresarial y la gestión financiera evidenciando las diferencias en las prácticas realizadas por las empresas más competitivas, y contrastándolas con las realizadas por las demás instituciones.

\section{RESULTADOS}

En la Tabla 1 se pretende reflejar la situación contable de las 252 MiPymes encuestadas, a pesar de que sólo el 1\% de los participantes llevan contabilidad formal; el $76 \%$ reconoce tener registros de los ingresos y egresos periódicos, mientras que el $23 \%$ indica que no lleva ningún tipo de registro del movimiento financiero de la empresa; estos datos coinciden con el tratamiento de los costos de producción, ya que sólo los 3 participantes que llevan la contabilidad, reconocieron que incluían las depreciaciones de las maquinarias y equipos en el cálculo del precio del producto; los demás no consideraban depreciaciones.

Tabla 1 Situación contable de las MiPymes

\begin{tabular}{|l|l|l|}
\hline RESPUESTA & NO. EMPRESAS & \multicolumn{1}{l|}{$\%$} \\
\hline Registros de Ingresos /Egresos & 191 & $76 \%$ \\
\hline Sin registros & 58 & $23 \%$ \\
\hline Contabilidad Formal & 3 & $1 \%$ \\
\hline Total & 252 & $100 \%$ \\
\hline
\end{tabular}

Se puede evidenciar en la Tabla 2, el 13\% de las empresas que fueron parte de la encuesta no cuenta con el Registro Único de Contribuyente, es decir, no han regularizado su estado tributario. 
Tabla 2 Situación tributaria de las MiPymes

\begin{tabular}{|l|l|l|}
\hline RESPUESTA & \multicolumn{1}{|l|}{ NO. EMPRESAS } & \multicolumn{1}{l|}{$\%$} \\
\hline No tiene RUC & 32 & $13 \%$ \\
\hline Tiene RUC & 220 & $87 \%$ \\
\hline Total & 252 & $100 \%$ \\
\hline
\end{tabular}

En la Tabla 3, se ha detallado el promedio de: la inversión requerida para la implementación de las empresas de los encuestados cuyo valor fue de \$ 25.500 , el flujo de efectivo generado anualmente fue $\$ 7.989$ y el ROE promedio 39\%, también muestra la desviación típica de 5.3\%. No está por demás indicar que estos valores no son exactos, sino un aproximado calculado por los participantes al momento de la encuesta; es necesario hacer esta aclaración dado que en la Tabla 1, se indica que el 23\% de los encuestados no lleva registros contables.

Tabla 3 Análisis de rentabilidad de las MiPymes

\begin{tabular}{|l|l|}
\hline \multicolumn{2}{|c|}{ PROMEDIO } \\
\hline Inversión total & 25.500 \\
\hline Flujo de efectivo & 7.989 \\
\hline ROE & $39 \%$ \\
\hline Desviación típica & $5,3 \%$ \\
\hline
\end{tabular}

La rentabilidad sobre el patrimonio se ha clasificado en 4 grupos principales en la Tabla 4, siendo el grupo mayoritario aquellas que tienen un ROE de 15\% o menos.

Tabla 4 Rentabilidad sobre inversión Pymes

\begin{tabular}{|l|l|l|}
\hline ROE & \multicolumn{1}{l|}{ NO. EMPRESAS } & \multicolumn{1}{l|}{$\%$} \\
\hline $15 \%$ o menos & 103 & $40,9 \%$ \\
\hline de $16 \%$ a $40 \%$ & 69 & $27,4 \%$ \\
\hline $41 \%$ al $100 \%$ & 67 & $26,6 \%$ \\
\hline más de $100 \%$ & 13 & $5,2 \%$ \\
\hline Total & 252 & 1 \\
\hline
\end{tabular}

Con relación al estado de endeudamiento, se puede observar que las fuentes de financiación son diversas, destacándose que el 51\% de los préstamos solicitados a los bancos, se hace por la modalidad de créditos de consumo con un $16.27 \%$ de interés anual; mientras que el $48 \%$ de los préstamos que reciben de agentes no bancarios, son créditos al 15\% mensual, con pagos diarios de capital e interés; se puede encontrar esta información más detallada en la Tabla 5. 
Tabla 5 Estado de endeudamiento de las MiPymes

\begin{tabular}{|c|c|c|}
\hline RESPUESTA & NO. EMPRESAS & $\%$ \\
\hline Préstamos bancarios & 89 & $35 \%$ \\
\hline Consumo $16,27 \%$ & 45 & $51 \%$ \\
\hline Empresarial 10,95\% & 23 & $26 \%$ \\
\hline Microcrédito minorista $25 \%$ & 21 & $24 \%$ \\
\hline Préstamos otras fuentes & 104 & $41 \%$ \\
\hline Pago mensual de intereses $10 \%$ & 31 & $30 \%$ \\
\hline *Pago diario capital interés 15\% mensual & 50 & $48 \%$ \\
\hline *Pago diario capital interés $20 \%$ mensual & 23 & $22 \%$ \\
\hline Sin préstamos & 59 & $23 \%$ \\
\hline Total & 252 & $100 \%$ \\
\hline
\end{tabular}

* Tasas de interés tomadas de las tasas de interés referenciales del BCE (2020)

En la tabla anterior, se podía observar que el 76\% de los participantes de la encuesta, es decir 193 empresas del grupo de las MiPymes, habrían solicitado préstamos a instituciones financieras y no financieras; en la Tabla 6, se puede observar cuál fue el destino de la financiación obtenida; más del 76\% de los préstamos se realizan para reponer material de trabajo y herramientas de uso diario, dejando esto en evidencia la falta de gestión financiera de las empresas para prever, el desgaste y la obsolescencia de los artículos de uso diario en la actividad económica.

Tabla 6 Destino de los préstamos que solicitan las MiPymes

\begin{tabular}{|l|l|l|}
\hline DESTINO DE LOS CRÉDITOS & \multicolumn{1}{c|}{ NO. EMPRESAS } & $\%$ \\
\hline Mejoras en la empresa & 11 & $5,7 \%$ \\
\hline Reposición de materiales y herramientas & 148 & $76,7 \%$ \\
\hline Gastos personales & 30 & $15,5 \%$ \\
\hline Otros & 4 & $2,1 \%$ \\
\hline Total & 193 & 1 \\
\hline
\end{tabular}

Indistintamente de la rentabilidad empresarial, 104 del total de encuestados solicitó préstamos a entes no regulados, el $79 \%$ de los participantes afirmaron haber intentado obtener financiación bancaria y haber recibido una negativa; mientras que el $21 \%$ basado en referencias, fue directamente a la fuente de financiación (Tabla 7).

Tabla 7 Motivaciones para elección de préstamos

\begin{tabular}{|c|c|c|}
\hline RESPUESTA & NO. EMPRESAS & $\%$ \\
\hline Negativa bancaria & 82 & $79 \%$ \\
\hline Fue directo a la fuente de financiación. & 22 & $21 \%$ \\
\hline Total & 104 & 1 \\
\hline
\end{tabular}


En la Tabla 8, muestra que solo el 35\% de los participantes destina una parte de sus ganancias en concepto de ahorros para emergencias e imprevistos.

Tabla 8 Nivel de ahorro de las MiPymes

\begin{tabular}{|l|l|l|}
\hline RESPUESTA & NO. EMPRESAS & \% \\
\hline $\mathrm{Si}$ & 88 & $35 \%$ \\
\hline No & 164 & $65 \%$ \\
\hline Total & 252 & 1 \\
\hline
\end{tabular}

\section{Establecimiento de relaciones}

Tomando como antecedente la Tabla 4, en esta sección se reflejará la situación contable, nivel y tipo de endeudamiento y ahorro de las empresas cuya Rentabilidad sobre Patrimonio sea mayor al promedio:

Tabla 8 Empresas participantes más rentables

\begin{tabular}{|l|l|l|l|l|l|l|}
\hline & ROE $>$ 40\% & \multicolumn{1}{l}{$\begin{array}{l}\text { REGISTRO } \\
\text { INGRESOS }\end{array}$} & PRÉSTAMOS & $\begin{array}{l}\text { PRÉSTAMOS } \\
\text { BANCOS }\end{array}$ & $\begin{array}{l}\text { TROS } \\
\text { PRÉSTAMOS }\end{array}$ \\
\hline $\begin{array}{l}\text { No. } \\
\text { EMPRESAS } \\
\text { PORCENTAJE }\end{array}$ & 80 & 74 & 76 & 69 & 7 & 78 \\
\cline { 2 - 7 } & $100 \%$ & $93 \%$ & $95 \%$ & $86 \%$ & $9 \%$ & $98 \%$ \\
\hline
\end{tabular}

En la Tabla 8 es necesario evidenciar que en lo referente a la fila de los valores del porcentaje estos corresponden al total de la población (80 empresas); del mismo modo, se debe destacar la positividad de estas cifras, ya que el 93\% lleva registros de ingresos y gastos, el 95\% ha trabajado con préstamos y estos han procedido de préstamos bancarios. La tasa de ahorro también es muy alentadora.

De la misma manera, a continuación se busca destacar la situación contable, nivel de endeudamiento, origen de la financiación y ahorro de las empresas cuyo ROE fue menor al del promedio.

Tabla 9 Empresas participantes menos rentables

\begin{tabular}{|l|l|l|l|l|l|l|}
\hline & ROE $>$ 40\% & \multicolumn{1}{l}{$\begin{array}{l}\text { REGISTRO } \\
\text { INGRESOS }\end{array}$} & PRÉSTAMOS & \multicolumn{1}{l}{$\begin{array}{l}\text { PRÉSTAMOS } \\
\text { BANCOS }\end{array}$} & $\begin{array}{l}\text { TROS } \\
\text { PRÉSTAMOS }\end{array}$ \\
\hline $\begin{array}{l}\text { No. } \\
\text { EMPRESAS } \\
\text { PORCENTAJE }\end{array}$ & 172 & 120 & 117 & 20 & 97 & 10 \\
\cline { 2 - 7 } & $100 \%$ & $70 \%$ & $68 \%$ & $12 \%$ & $56 \%$ & $6 \%$ \\
\hline
\end{tabular}

La Tabla 9, muestra notables diferencias, comparada con la Tabla 8, ya que solo un $70 \%$ de este tipo de empresas tiene un registro de ingresos y egresos; y a pesar de que el nivel de endeudamiento es menor, más del 56\% del total 
de la población ha solicitado préstamos a entidades no reguladas; el nivel de ahorro es totalmente opuesto a la tabla 8 , pues solo un $6 \%$ de los encuestados tiene ahorros para imprevistos.

Con la información obtenida, se ha realizado un modelo relacional en el que se pueda evidenciar de manera gráfica las diferencias encontradas entre las empresas consideradas "más rentables" y el resto de empresas.

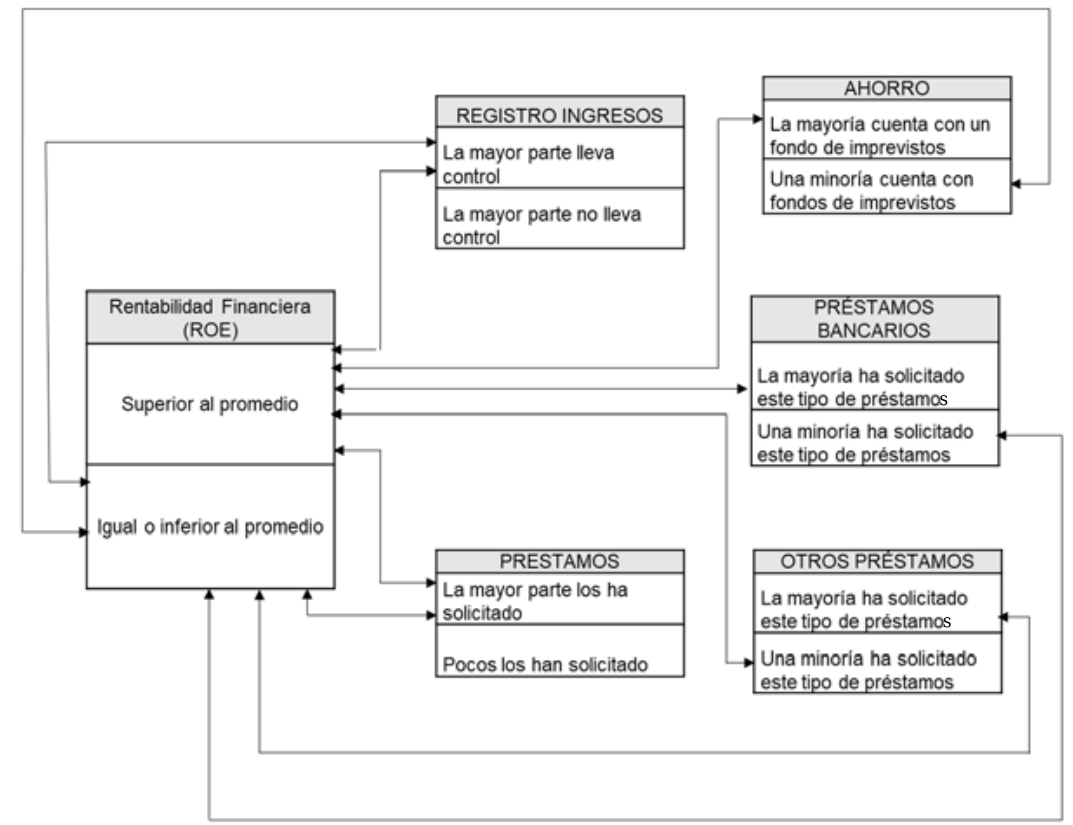

Figura 1. Modelo relacional de las MiPymes de la ciudad de Machala

Como se puede apreciar en la Ilustración 1 se encuentran similitudes entre el control de ingresos y gastos, y el endeudamiento, las diferencias más notables se encuentran en las fuentes de financiación, las empresas con una rentabilidad superior al promedio han recurrido a préstamos en instituciones bancarias, mientras que la mayoría de las empresas con una rentabilidad inferior al promedio han recurrido a financiamiento con organizaciones que se encuentran al margen del sistema financiero con elevadas tasas de intereses (Ver Tabla 5); también se puede establecer una clara diferencia entre la proporción de personas que cuentan con ahorros y/o fondos para imprevistos.

\section{CONCLUSIONES}

El desconocimiento de aspectos contables básicos hace que menos el 1\% de los participantes consideren rubros importantes en sus costos operativos, como depreciaciones o tasas de intereses; como resultado de los límites y trabas encontradas en la búsqueda de financiación bancaria de algunos microempresarios, se ven obligados a recurrir a otras formas de financiamiento 
no legales, con intereses exagerados (ver Tabla 5); el impacto de esta mala práctica de gestión financiera no sólo disminuye la competitividad de las empresas al aumentar sus costos, y de forma consecuente el precio final de los productos, sino que reduce sus ganancias, disminuye las posibilidades de invertir para ser más competitivos y también se ve reflejada en su nivel de ahorro.

Con lo expuesto en el párrafo anterior, es posible establecer una relación directa entre la gestión financiera de las MiPymes de la ciudad de Machala y la competitividad de las mismas, ya que entre mejor es su gestión financiera es más rentable y por lo tanto más competitiva; se debe destacar también la importancia del endeudamiento, el cual es saludable para la empresa desde un punto de vista financiero siempre que se analice la conveniencia de las tasas de intereses.

\section{Recomendaciones}

Se considera necesario incluir las siguientes recomendaciones para las MiPymes:

- Es importante mantener un control de ingresos y egresos, pero los mismos no deben tener como destino exclusivo el cumplimiento de obligaciones con el Servicio de Rentas internas, este debe servir para la toma de decisiones y planificación estratégica.

- Aunque las depreciaciones no son una obligación exigible, deben ser consideradas en el cálculo de costos de producción; se aconseja que este rubro sea depositado en una cuenta bancaria en donde cumplirá tres objetivos: será parte de la reserva en caso de imprevistos, generará intereses y el flujo constante de capitales facilitará el acceso a financiación bancaria en un futuro.

- Otro aspecto necesario es la elaboración de un presupuesto que permita prever los requerimientos de efectivo, como una forma de evitar caer en la tentación de "préstamos emergentes" por parte de instituciones no reguladas.

Al finalizar, se busca concientizar a las MiPymes de separar las cuentas personales de las cuentas de la empresa, esta práctica dificulta el control financiero del negocio. 


\section{REFERENCIAS BIBLIOGRÁFICAS}

Águila, A., \& Tolamatl -Michcol, J. (2016). Análisis de la innovación, la tecnología, los recursos humanos y la infraestructura como impulsores de la competitividad manufacturera del sector autopartes. Comparación y recomendaciones para el estado de Tlaxcala. Bloomington: Palibrio.

Azeez, K., Kadhim, H. , \& Kadhim, A. (2020). The role of integration between enterprise resource planning and attribute based costing for supporting economic cost management in tourism companies. African Journal of Hospitality, Tourism and Leisure, 1-10. Recuperado el 04 de 08 de 2020, de https://www.ajhtl.com/uploads/7/1/6/3/7163688/article_16_vol_9_2_2020_ iraq.pdf

Banco Central del Ecuador. (16 de 02 de 2021). TASAS DE INTERÉS ACTIVAS EFECTIVAS VIGENTES PARA EL SECTOR FINANCIERO PRIVADO, PÚBLICO Y, POPULAR Y SOLIDARIO. Obtenido de https://contenido.bce.fin. ec/docs.php?Path=/documentos/Estadisticas/sectormonfin/tasasinteres/ Indice.htm

Bermeo, R., \& Bermeo, A. (2005). LAS DIRECTRICES DEL COSTO COMO FUENTES DE VENTAJAS COMPETITIVAS. Estudios Gerenciales(94), 81106. Https://www.redalyc.org/pdf/212/21209405.pdf

Campion, A., Ekka, R. , \& Wenner, M. (2012). Las tasas de interés y sus repercusiones en las microfinanzas en América Latina y el Caribe. Banco Interamericano de Desarrollo. Obtenido de https://publications.iadb.org/ publications/spanish/document/Las-tasas-de-inter\%C3\%a9s-y-sus-repercusiones-en-las-microfinanzas-en-Am\%C3\%a9rica-Latina-y-el-Caribe.pdf

Choi, S. (2021). Currency Valuation, Export Competitiveness, and Firm Profitability: Evidence from Bangladeshi Firm-Level Data. Journal of Asian Finance, Economics and Business, 8(1), 061-069. Doi: http://doi. org/10.13106/jafeb.2021.vol8.no1.061

Corporación Universidad de Asturias. (2014). Conceptos básicos para las empresas, Competitividad, Eficiencia y Eficacia. Asturias. Https://cld.bz/ TE6yL0w\#2/z

Deming, W. (2018). Out of the Crisis. Quality, Productivity and Competitive Position. MIT Press; Edición: Reissue.

Dzhalilova, N., \& Meza-Godoy, A. (2020). Formation of the competitive potential of the agricultural territories. Entrepreneurship and Sustainability Issues, 7(3), 1921-1936. Https://doi.org/10.9770/jesi.2020.7.3(32)

García-Magaña, R., Vargas-Hernández, J. (2014). INCUBADORAS DE EMPRESAS COMO ALIADAS EN LA REDUCCIÓN DE COSTOS DE TRANSACCIÓN DE LOS EMPREDEDORES EN MÉXICO. Revista Reuna, 5-14. Http://biblio.ecotec.edu.ec/revista/edicion7/articulo-1.pdf

Ha, V. , \& Pham, H. (2021). Determinants of Bank Profitability in Vietnam: An Empirical Lasso Study. Studies in Computational Intelligence, 898, 397406. Doi: http://doi.org/10.1007/978-3-030-48853-6_28 
Haro, A., Gálvez, M., Sáez, A., \& Caba, C. (2017). EL ROL DEL CONSEJO DE ADMINISTRACIÓN EN LA ÉTICA EMPRESARIAL EN PAÍSES DE LATINOAMÉRICA. Revista de Administração de Empresas, 57(5). Doi: https:// doi.org/10.1590/s0034-759020170502

Jewell, P., Reading, J., Clarke, M., \& Kippist, L. (2020). Information skills for business acumen and employability: A competitive advantage for graduates in Western Sydney. Journal of Education for Business, 95(2), 88-105. Https://doi.org/10.1080/08832323.2019.1610346

Karlsson, N. (2019). Business models and business cases for financial sustainability: Insights on corporate sustainability in the Swedish farm-based biogas industry. Sustainable Production and Consumption, 18, 115-129. Https://doi.org/10.1016/j.spc.2019.01.005

Kwak, E., Kim, G., \& Yoo, J. (2011). Network operation cost model to achieve efficient operation and improving cost competitiveness. 13th International Conference on Advanced Communication Technology (pp. 1107-1112). San Diego, CA, USA: IEEE. Https://ieeexplore.ieee.org/document/5746001/citations\#citations

Ley de Compañías. (20 de mayo de 2014). Registro Oficial 312 de 05-nov1999. Quito. Obtenido de https://www.supercias.gob.ec/bd_supercias/ descargas/lotaip/a2/Ley-Cias.pdf

LORTI, L. O. (17 de noviembre de 2004). Artículo 160. Obtenido de https://www.sri.gob.ec/bibliotecaportlet/descargar/cbac1cfa-7546-4bf4-ad32-c5686b487ccc/20151228+LRTI.pdf

Liu, C. (2017). International competitiveness and the fourth industrial revolution. Entrepreneurial Business and Economics Review, 5(4), 111-133. Https://doi.org/10.15678/EBER.2017.050405

Modigliani, F., \& Miller, M. (1963). Corporate income taxes and the cost of capital: A correction. The American Economic Review, 53(3), 433-443. Retrieved from https://www.jstor.org/stable/1809167

Pacheco, C. (2015). Las finanzas y los negocios internacionales, su impacto en la competitividad. Mexico: Instituto Mexicano de contadores Públicos.

Porter, M. (2017). Ser competitivo. Deusto.

Ricardo, D. (2003). Principios de Economía Política y Tributación. Pirámide.

Rivera, J. (2002). Teoría sobre la estructura de capital. Estudios Gerenciales, 18(84), 31-59. Retrieved from http://www.scielo.org.co/scielo. php?Script=sci_abstract\&pid=S0123-59232002000300002\&lng=en\&n$\mathrm{rm}=\mathrm{iso \& t}$ tlng $=\mathrm{es}$

Rubio, A., \& Aragón, A. (2007). Análisis de los efectos empresa e industria en las PYME murcianas. Cuadernos de Ciencias Económicas y Empresariales, ISSN 0211-4356, № 53, 2007, pags. 83-105, 83-105. Http://cuadernos. uma.es/pdfs/pdf645.pdf

Saavedra, M., Milla, S., \& Tapia, B. (2013). La competitividad de la Mipyme en el nivel micro: El caso de Querétaro, México. En-Contexto Revista de Investigación en Administración, Contabilidad, Economía y Sociedad, 175-203. Https://www.redalyc.org/jatsrepo/5518/551857515008/html/index.html 
Sánchez-Gutiérrez, J., Cabanelas, P., Lampón, J. F., \& González-Alvarado, T. E. (2019). The impact on competitiveness of customer value creation through relationship capabilities and marketing innovation. Journal of Business and Industrial Marketing, 34(3), 618-627. Https://doi.org/10.1108/ JBIM-03-2017-0081

Smith, A. (2011). La riqueza de las naciones: libros I-II-III y selección de los libros IV y V. Alianza Editorial.

Solleiro, J., \& Castañón, R. (2005). Competitiveness and innovation systems: The challenges for Mexico's insertion in the global context. Technovation, 1059-1070. Https://doi.org/10.1016/j.technovation.2004.02.005

SRI, S. D. (12 de 11 de 2020). Servicio de Rentas Internas. Obtenido de https://www.sri.gob.ec/web/guest/catastros

Suñol, S. (abril-junio de 2006). Aspectos teóricos de la competitividad. Ciencia y Sociedad, XXXI(2), 179-198. Https://www.redalyc.org/pdf/870/87031202. pdf

Staníčková, M., \& Melecký, L. (2020). Threats and challenges for the competitiveness of the european union: What are the perspectives for cohesion among the member states? In R. Moldovan (Ed.), The European Union: Policies, Perspectives and Politics (pp. 63-84). NOVA. Https://ec.europa. eu/regional_policy/sources/policy/what/territorial-cohesion/territorial_ state_and_perspective_2011.pdf

\section{Cumbres}

